\title{
Tailoring the Interaction Strength between Gold Particles and Silica Thin Films via Work Function Control
}

\author{
Umberto Martinez, ${ }^{1}$ Jan-Frederik Jerratsch, ${ }^{2}$ Niklas Nilius, ${ }^{2, *}$ Livia Giordano, ${ }^{1}$ \\ Gianfranco Pacchioni, ${ }^{1, \dagger}$ and Hans-Joachim Freund ${ }^{2}$ \\ ${ }^{1}$ Dipartimento di Scienza dei Materiali, Università di Milano-Bicocca, via Cozzi, 53 - 20125 Milano, Italy \\ ${ }^{2}$ Department of Chemical Physics, Fritz-Haber Institut der Max-Planck Gesellschaft, Faradayweg 4-6, D-14195 Berlin, Germany
}

(Received 26 March 2009; published 27 July 2009)

\begin{abstract}
The possibility to modify the adsorption properties of a porous silica/Mo(112) film by controlling its work function has been studied by a combined STM and density-functional theory approach. While the original film is inert towards metal adsorption, Au atoms and clusters can be stabilized on the surface after $\mathrm{Li}$ doping. The $\mathrm{Li}$ atoms penetrate the topmost silica layer and bind $\mathrm{as}^{+}{ }^{+}$cations at the metal-oxide interface, thereby reducing the oxide work function. This induces a charge transfer into Au adatoms, which in turn enables strong Au-silica interaction mediated by a polaronic distortion of the oxide lattice.
\end{abstract}

The stabilization of highly dispersed metal particles on oxide supports is of decisive importance for applications in heterogeneous catalysis [1] and for the development of sensor, magnetic storage, and optoelectronical devices [2-4]. High catalytic activity, for example, is often restricted to small particle sizes and disappears when the admaterial adapts bulklike properties. The anchoring of metal deposits to inert, wide-gap oxides usually involves surface defects that offer substantially higher binding energies than the regular surface [5]. In this work, an alternative stabilization mechanism is presented that is independent of defect-mediated adsorption and exploits the possibility to tailor the charge state of particles on thin oxide films on metal supports. Charged adspecies are able to activate new interaction schemes with the oxide surface, such as Coulomb attraction towards oppositely charged oxide ions, polaronic distortions of the oxide lattice, and interactions with image charges formed in the metal underneath [6]. They consequently experience an enhanced binding strength to the oxide support with respect to their neutral counterparts. The charging phenomenon originates from electron tunneling between low-lying affinity levels in the adsorbate and the metal underneath, whereby the direction of the electron flow is governed by the work function $\Phi$ of the metal-oxide system [7]. Charge-mediated binding mechanisms have been revealed by theory and experiment for gold on different thin film systems, such as $\mathrm{MgO} / \mathrm{Ag}(001)$ [6,8-10], alumina/ $\mathrm{NiAl}(110)$ [11], and $\mathrm{FeO} / \mathrm{Pt}(111)$ [12,13]. Whereas in the first two examples negative charging of the Au takes place due to the low metal-oxide work function, the Au becomes cationic in the latter case, reflecting the high $\Phi$ value of the $\mathrm{FeO} / \mathrm{Pt}$ system.

Charging and stabilization of adparticles does not occur spontaneously on every oxide film. On silica/Mo(112) films, for example, Au remains neutral and is unable to bind to defect-free oxide patches $[14,15]$. Charge transfer is prevented in this case by the negative surface dipole associated with the silica growth, which raises $\Phi$ and acts against an electron flow into the gold [7]. The oxideinduced dipole might, however, be reversed by doping the system with electropositive species, e.g., alkali atoms. Recent calculations have shown that $\mathrm{Li}, \mathrm{Na}$, or $\mathrm{K}$ easily ionizes on the silica film, thereby lowering the work function and promoting electron transfer into Au species [16]. The smaller alkali atoms, $\mathrm{Li}$ and $\mathrm{Na}$, even penetrate the porous silica film and bind to the metal-oxide interface. Also in those subsurface sites, the alkalis reduce the work function and are additionally protected against chemical processes taking place at the oxide surface. This joint theoretical-experimental study demonstrates that a Lidoped silica/Mo(112) film is indeed able to anchor $\mathrm{Au}$ particles even when no surface defects are present.

The experiments are performed with a custom-build ultrahigh vacuum STM operated at $10 \mathrm{~K}$. Electronic properties of the sample are detected with differential conductance $(d I / d V)$ spectroscopy, providing a measure for the local density of states (LDOS) [17]. The silica film is prepared by depositing 1.2 monolayer $\mathrm{Si}$ in $1 \times$ $10^{-7} \mathrm{mbar}_{2}$ onto an oxygen precovered $\mathrm{Mo}(112)$ surface and annealing the sample to $1200 \mathrm{~K}$. It consists of a single layer of $\mathrm{SiO}_{4}$ tetrahedrons with three of the $\mathrm{O}$ atoms forming $\mathrm{Si}-\mathrm{O}-\mathrm{Si}$ bridges in the plane and the fourth one binding to the Mo underneath [18]. The tetrahedrons are arranged in a honeycomb structure made of six-membered -Si-O- rings with $5 \AA$ diameter that give access to nanopores at the metal-oxide interface. The Li atoms are introduced from a commercial Li dispenser at $100 \mathrm{~K}$, while $\mathrm{Au}$ is deposited at $20 \mathrm{~K}$ from a gold-plated tungsten filament. Density-functional theory (DFT) calculations are performed with the generalized gradient approximation as implemented in the VASP code [19], using the PW91 exchange-correlation functional [20] and a plane wave basis set (energy cutoff of $400 \mathrm{eV}$ ). The electron-ion 
coupling is described by the projector augmented wave method [21]. Supercells of various sizes are constructed to model the interaction of $\mathrm{Li}$ and $\mathrm{Au}$ with the silica/Mo(112) film. The largest $(6 \times 4)$ cell had hereby a composition of $\mathrm{Mo}_{96} \mathrm{Si}_{24} \mathrm{O}_{60}$. STM images are simulated using the TersoffHamann approach [17].

Already single $\mathrm{Li}$ atoms are identified on the surface due to their distinct bias-dependent contrast in the STM [Fig. 1(a)]. At low imaging bias, two Li-induced features of $0.15 \AA$ apparent height are distinguished, namely, starlike protrusions (termed $\mathrm{Li}_{1}$ ) and short [1110]-oriented bars $\left(\mathrm{Li}_{1}{ }^{*}\right)$. Both configurations occur in comparable numbers and are statistically distributed on the surface. They are not compatible with $\mathrm{Li}$ adatoms bound atop the oxide film, but seem to reflect distinct structural elements of the silica network that become emphasized upon $\mathrm{Li}$ adsorption. At a sample bias above $2.0 \mathrm{~V}$, the Li-induced features transform into bright, circular protrusions of $\sim 0.5 \AA$ height [Fig. 1(a), inset]. A comparable bias-dependent contrast was earlier observed for Pd atoms, which were found to penetrate the topmost oxide layer and bind to the silica/Mo interface [14,15]. A similar behavior is now revealed for $\mathrm{Li}$, as demonstrated by the DFT calculations. The Li atoms are able to pass the silica nanopores with a barrier of $0.3 \mathrm{eV}$ and bind to interfacial sites located either below the center of an oxide ring $\left(\mathrm{Li}_{1}: E_{B}=2.43 \mathrm{eV}\right)$ or below a [110]-oriented Si-O-Si bridge $\left(\mathrm{Li}_{1}{ }^{*}: E_{B}=2.41 \mathrm{eV}\right)$. The two adsorption geometries are associated with an unequal level of hybridization between the empty Li $2 s$ and the $\mathrm{O}$ $2 p$ states and consequently lead to a different appearance of both $\mathrm{Li}$ species in simulated as well as experimental STM images [Figs. 1(b) and 1(c)].

According to the DFT, Li becomes cationic in its interfacial binding site and donates a full electron to the Mo support. As a result, the work function $\Phi$ around the posi-

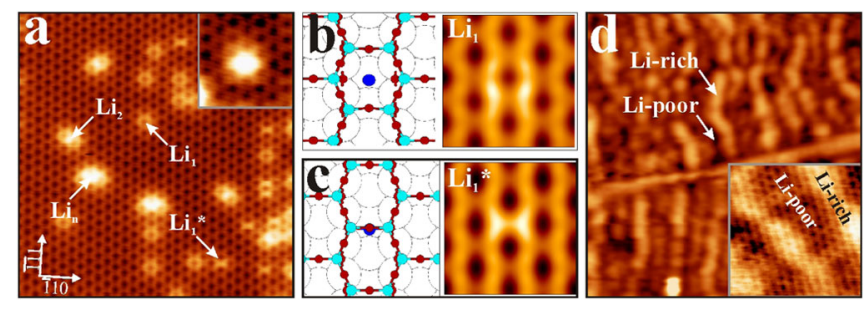

FIG. 1 (color online). (a) Single $\mathrm{Li}$ atoms embedded into silica/Mo(112) $\left(1.0 \mathrm{~V}, 15 \times 15 \mathrm{~nm}^{2}\right)$. Two binding configurations $\left(\mathrm{Li}_{1}\right.$ and $\left.\mathrm{Li}_{1}{ }^{*}\right)$ are observed in the experiment. The inset shows a $\mathrm{Li}_{1}$ species imaged at $2.8 \mathrm{~V}$ sample bias. (b), (c) Structure models and simulated STM images for both Li species [O, small dark (red) spheres; $\mathrm{Si}$, small light (cyan) spheres; and Li, large blue spheres]. (d) STM image of silica/Mo(112) with a mean coverage of $0.7 \mathrm{Li}^{+}$ions per silica pore $\left(1.0 \mathrm{~V}, 25 \times 25 \mathrm{~nm}^{2}\right)$. The vertical bright lines are Li-rich islands, whereas the horizontal line in the image center is a $\mathrm{Li}$ filled domain boundary. The inset shows the same surface region with enhanced resolution $\left(7 \times 7 \mathrm{~nm}^{2}\right)$. tive dopant decreases by $0.3 \mathrm{eV}$, as calculated for an effective coverage of one Li atom per four silica pores. Further $\mathrm{Li}$ incorporation leads to a continuous $\Phi$ reduction, reaching 1.1 and $1.7 \mathrm{eV}$ for one and two $\mathrm{Li}$ ions per silica pore, respectively [Fig. 2(c)]. The Li-induced work function change is experimentally determined by probing the onset of the silica bands with $d I / d V$ spectroscopy. Because of the positive dipole created by the $\mathrm{Li}^{+}$ions, the vacuum energy experiences a downward step at the interface that also shifts the oxide electronic states to lower energies. In particular, the conduction band onset, being identified as a kink in the $d I / d V$ curves, moves from 2.8 to $2.4 \mathrm{~V}$ with increasing Li dosage and broadens considerably [Fig. 2(a)] [22]. A similar behavior is revealed for the calculated band onsets [Fig. 2(b)]. Absolute band positions and the slope of the work function as a function of Li coverage slightly differs in experiment and theory due to the known deficiency of DFT to reproduce gap sizes of insulating materials. However, the observed downshift of the band onset upon $\mathrm{Li}$ exposure provides clear evidence for the work function decrease predicted by DFT [Fig. 2(c)].

At high exposure, the spatial distribution of $\mathrm{Li}$ at the metal-oxide interface becomes strongly inhomogeneous, as the dopants form islands that are 4-5 pores wide and elongated along the [111] direction [Fig. 1(c)]. Whereas the $\mathrm{Li}$ coverage within the stripes reaches one $\mathrm{Li}^{+}$per silica pore, the oxide regions in between are $\mathrm{Li}$ poor $\left(<0.5 \mathrm{Li}^{+}\right.$per pore $)$and show substantially higher $\Phi$ values. The spatial ordering of the interfacial $\mathrm{Li}$ is the consequence of a modified penetration probability of the alkali atoms in the presence of subsurface $\mathrm{Li}^{+}$and the distinct screening response of the Mo support to the charge impurities. A detailed analysis of the phenomenon is given in a forthcoming paper.

The Li doping induces a dramatic change in the adsorption behavior of the silica/Mo film with respect to gold, as shown in Fig. 3. Prior to $\mathrm{Li}$ incorporation, Au atoms are
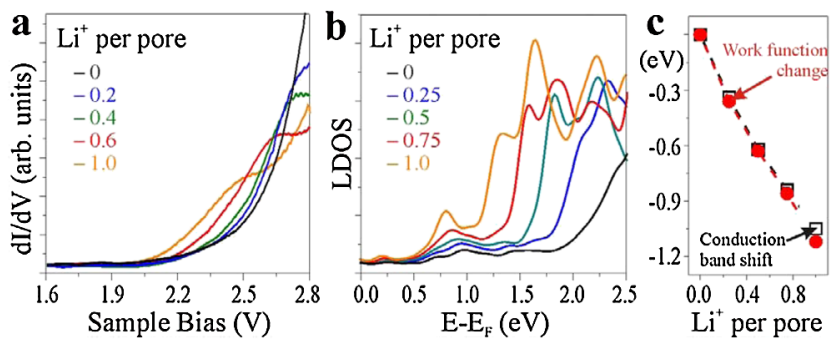

FIG. 2 (color online). (a) Differential conductance spectra taken on silica/Mo(112) for different amounts of incorporated Li. The kink marks the onset of the silica conduction band, which gradually shifts to lower energies with increasing $\mathrm{Li}$ exposure. (b) Calculated LDOS of silica/Mo(112) in the region of the conduction band for various Li coverages. (c) Correlation between work function decrease and conduction band shift of silica/Mo(112) calculated as a function of $\mathrm{Li}$ coverage. 

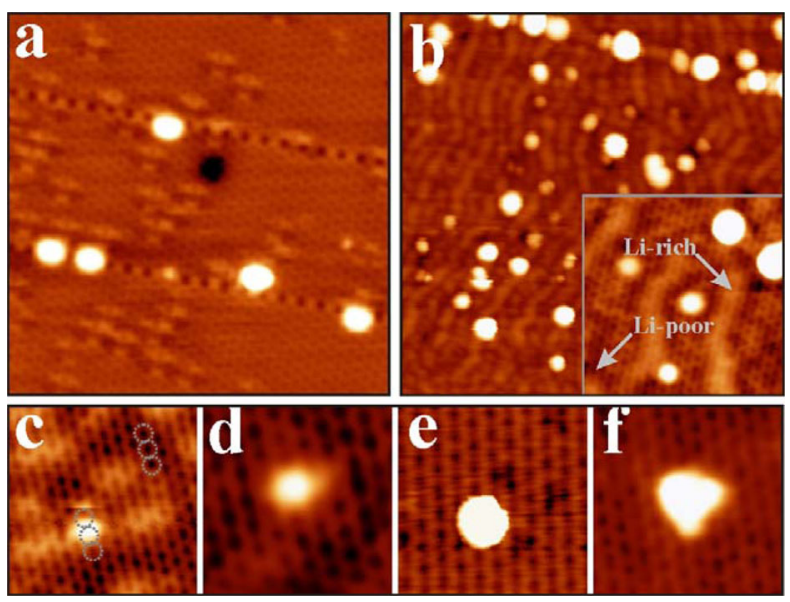

FIG. 3 (color online). (a) Pristine $\left(2.0 \mathrm{~V}, 20 \times 20 \mathrm{~nm}^{2}\right)$ and (b) Li-doped silica film after deposition of 0.1 monolayer $\mathrm{Au}\left(2.0 \mathrm{~V}, 50 \times 50 \mathrm{~nm}^{2}\right)$. Whereas $\mathrm{Au}$ attaches exclusively to domain boundaries for the undoped film, it binds to defect-free oxide patches after incorporation of $0.7 \mathrm{Li}^{+}$ions per silica pore. The inset in (b) shows an oxide region with enhanced resolution $\left(0.6 \mathrm{~V}, 11 \times 11 \mathrm{~nm}^{2}\right)$. (c) Single $\mathrm{Au}$ atom on Li-doped silica. The adatom does not sit above a pore, but between two hexagonal rings as marked by the dashed circles. (d)-(f) Au clusters with increasing size stabilized on Li-doped silica. The $\mathrm{Au}$ growth follows the Vollmer-Weber regime and leads to the formation of 3D particles.

unable to bind to the defect-free film and rapidly diffuse on the surface until they become trapped at domain boundaries between two oxide patches [Fig. 3(a)]. Along these line defects, the six-membered-Si-O- rings are replaced by eight-membered entities, whose diameter is sufficiently large to accommodate the Au. Those defect-bonded adatoms serve as nuclei for the growth of three-dimensional (3D) particles, decorating the domain boundaries [Fig. 3(a)] [23]. This adsorption behavior completely changes after $\mathrm{Li}$ doping, when $\mathrm{Au}$ atoms and clusters also appear on the defect-free oxide terraces away from the domain boundaries [Fig. 3(b)]. At low Au exposure, mainly single adatoms with spherical shape and $\sim 0.8 \AA$ height are detected [Fig. 3(c)]. The absence of starlike shapes in this case, being the fingerprint for an embedded species as demonstrated for Li and Pd [15], suggests an $\mathrm{Au}$ atom location above the oxide surface. This conclusion is supported by the fact that single adatoms are found on top of the -Si-O- network and not above the nanopores. In most cases, the $\mathrm{Au}$ atoms and clusters attach to the Li-rich stripes with their lower work function, but occasionally bind to Li-poor regions as well [Fig. 3(b)]. With increasing Au coverage, 3D particles develop on the modified surface with an average density of $2.5 \times 10^{12} \mathrm{~cm}^{-2}$. The deposits adopt mainly prolate shapes with a large height to diameter ratio of $\sim 0.3$, but sometimes exhibit triangular and hexagonal base planes [Figs. 3(e) and 3(f)]. Whereas Au monomers do not show conductance peaks that would indicate tunneling into defined atomic orbitals, a number of $d I / d V$ maxima are revealed for larger particles, suggesting the formation of quantum well states in the confined electronic system (not shown).

The stabilization of $\mathrm{Au}$ atoms and clusters on $\mathrm{Li}$-doped silica/Mo(112) films is fully consistent with the predictions derived from the DFT calculations. For a mean coverage of one $\mathrm{Li}^{+}$per pore, the Au binds with $1.33 \mathrm{eV}$ to the $\mathrm{O}$ atoms in [110]-oriented $\mathrm{Si}-\mathrm{O}-\mathrm{Si}$ bridges, which is an order of magnitude stronger than to the undoped film [Fig. 4(a)]. According to the computed LDOS, the adatom has a completely filled Au $6 s$ orbital and zero spin density, which indicates the transfer of one extra electron from the support into the $\mathrm{Au}$ atom [Fig. 4(c)]. The formation of anionic $\mathrm{Au}$ species is confirmed by the Bader charge value of $-0.8|e|$ and by the strong polaronic distortion of the silica in response to the anionic perturbation [Fig. 4(b)]. The lattice distortion is hereby essential to stabilize the charge state of gold, and only possible for those $\mathrm{O}$ atoms that span the [111]-oriented troughs of the Mo(112) surface. There are two other local minima for binding the Au atom, namely, above the center of a silica ring $\left(E_{B}=0.34 \mathrm{eV}\right)$ and above a [111]-oriented Si-O-Si unit running atop a protruding Mo row $\left(E_{B}=0.18 \mathrm{eV}\right)$ [Fig. 4(a)]. However, as a significant lattice distortion is impossible at both sites, the charge transfer from the Mo support into the $\mathrm{Au}$ is suppressed and the interaction energy remains small [Fig. 4(c)]. A polaronic distortion has also been found for Au adsorption on $\mathrm{NaCl} / \mathrm{Cu}(111)$ [24] and $\mathrm{MgO} / \mathrm{Ag}(100)$ thin films [7], and seems to be of general importance to stabilize charged species on ionic films. Not surprisingly, the charging of $\mathrm{Au}$
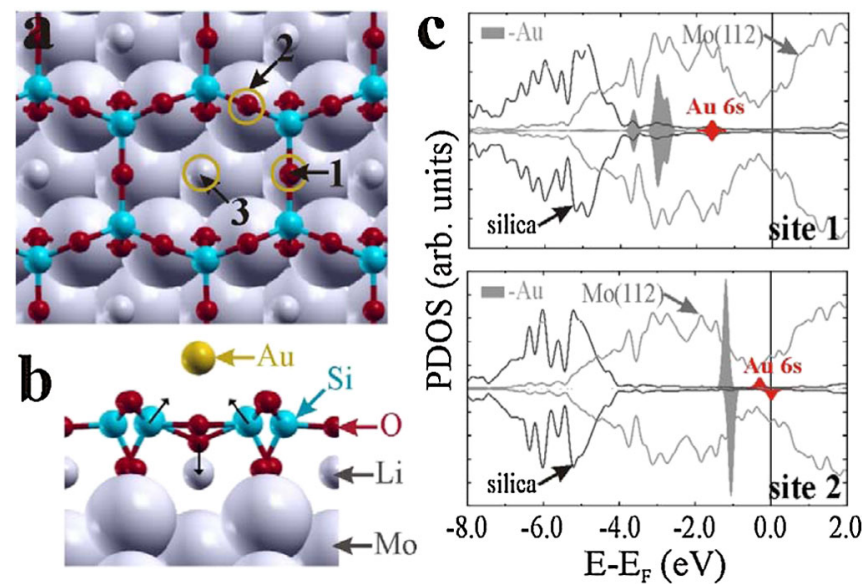

FIG. 4 (color online). (a) Structure model showing potential $\mathrm{Au}$ adsorption sites on silica/Mo(112) doped with one $\mathrm{Li}^{+}$per pore: (1) on the $\mathrm{O}$ atom of a 110 -oriented $\mathrm{Si}-\mathrm{O}-\mathrm{Si}$ bridge that suspends two Mo rows, (2) on a Si-O-Si bridge on top of a Mo row, and (3) above a nanopore filled with an interfacial $\mathrm{Li}$ atom. (b) Schematic representation of the polaronic distortion around the adsorbed $\mathrm{Au}$ atom on site 1. (c) Partial density of states projected onto an $\mathrm{Au}$ atom adsorbed on position 1 (top) and 2 (bottom). 
atoms is more efficient for a low silica/Mo work function and hence for higher Li coverage. The formation of anionic gold prevails, however, for a minimum Li content of one atom per four silica pores, although the $\mathrm{Au}$ binding energy to [110]-oriented Si-O-Si units decreases to $0.48 \mathrm{eV}$ in this case.

The strongly bound $\mathrm{Au}^{-}$species on a Li-doped silica/Mo(112) film acts as a nucleation center for particle growth. The resulting deposits adopt pronounced 3D shapes (see Fig. 3), which indicates a rather small overall Au-silica adhesion. Also DFT calculations demonstrate that an $\mathrm{Au}_{20}$ cluster with pyramidal shape is by $0.97 \mathrm{eV}$ more stable than its flat-lying 2D counterpart. Although this energy difference is smaller than between the respective gas-phase isomers $(1.9 \mathrm{eV})$, the $\mathrm{Au}-\mathrm{Au}$ and not the Au-support interaction dictates the growth regime of $\mathrm{Au}$ particle on the silica film. This finding is easily explained by the fact that strong Au-silica bonds are only formed to oxygen atoms in the [110]-oriented Si-O-Si bridges, while on adjacent surface sites the amount of charge transfer and hence the $\mathrm{Au}$ binding strength are much smaller. The Au particles are thus pinned to distinct positions in the silica film and a true $2 \mathrm{D}$ interaction is absent. On $\mathrm{MgO} / \mathrm{Ag}(001)$ thin films in contrast, negative charging takes place for every $\mathrm{Au}$ atom in contact with the $\mathrm{MgO}[6,9]$, and single-layered $\mathrm{Au}$ islands develop due to this spatially homogenous adhesion [10].

In conclusion, we have shown with a combined theoretical-experimental approach that the adsorption properties of silica/Mo(112) thin films can be tailored by modifying the work function of the system via doping. Exposing the silica surface to $\mathrm{Li}$ vapor results in the formation of $\mathrm{Li}^{+}$cations at the metal-oxide interface that produce a strong positive dipole. The associated work function decrease renders the silica film reactive and enables the stabilization of $\mathrm{Au}$ atoms and clusters on the originally inert oxide surface. The underlying adsorption mechanism is related to a charge transfer from the Mo metal into the Au atoms, which initiates a polaronic distortion of the oxide lattice. The strongly bound $\mathrm{Au}^{-}$species constitute the seeds for further cluster growth. Our study demonstrates the possibility to anchor adsorbates on oxide thin films by controlling their charge state. While the present results are referred to silica films, the procedure might be applied to other oxide materials, providing a general method to design the properties of metal-oxide interfaces.

The work has been supported by the COST Action D41. Part of the computing time was provided by the Barcelona Supercomputing Center (BSC-CNS).
*Corresponding author.

nilius@fhi-berlin.mpg.de

Corresponding author. gianfranco.pacchioni@unimib.it

[1] Handbook of Heterogeneous Catalysis, edited by G. Ertl, H. Knoezinger, F. Schueth, J. Weitkamp (Wiley-VCH, Weinheim, 2008), 2nd ed.

[2] D. Q. Yang, M. Meunier, and E. Sacher, J. Appl. Phys. 95, 5023 (2004).

[3] J. Bansmann et al., Surf. Sci. Rep. 56, 189 (2005); W. Schindler, D. Hofmann, and J. Kirschner, J. Appl. Phys. 87, 7007 (2000).

[4] C. Noguez, Opt. Mater. (Amsterdam) 27, 1204 (2005).

[5] V.E. Heinrich and P. A. Cox, The Surface Science of Metal Oxides (Cambridge University Press, Cambridge, England, 1994).

[6] G. Pacchioni, L. Giordano, and M. Baistrocchi, Phys. Rev. Lett. 94, 226104 (2005).

[7] L. Giordano, F. Cinquini, and G. Pacchioni, Phys. Rev. B 73, 045414 (2006).

[8] P. Frondelius, H. Häkkinen, and K. Honkala, Phys. Rev. B 76, 073406 (2007).

[9] D. Ricci, A. Bongiorno, G. Pacchioni, and U. Landman, Phys. Rev. Lett. 97, 036106 (2006).

[10] M. Sterrer et al., Phys. Rev. Lett. 98, 096107 (2007); M. Sterrer, T. Risse, M. Heyde, H.P. Rust, and H. J. Freund, Phys. Rev. Lett. 98, 206103 (2007).

[11] N. Nilius, V. G. Pirovano, V. Bradzova, M. Kulawik, J. Sauer, and H.-J. Freund, Phys. Rev. Lett. 100, 096802 (2008).

[12] N. Nilius, E. D. L. Rienks, H. P. Rust, and H. J. Freund, Phys. Rev. Lett. 95, 066101 (2005).

[13] L. Giordano et al., Phys. Rev. Lett. 101, 026102 (2008).

[14] L. Giordano, A. Del Vitto, and G. Pacchioni, J. Chem. Phys. 124, 034701 (2006).

[15] S. Ulrich et al., Chem. Phys. Chem. 9, 1367 (2008).

[16] U. Martinez, L. Giordano, and G. Pacchioni, J. Chem. Phys. 128, 164707 (2008).

[17] J. Tersoff and D. R. Hamann, Phys. Rev. Lett. 50, 1998 (1983).

[18] J. Weissenrieder et al., Phys. Rev. Lett. 95, 076103 (2005).

[19] G. Kresse and J. Hafner, Phys. Rev. B 47, 558 (1993); G. Kresse and J. Furthmüller, Phys. Rev. B 54, 11169 (1996).

[20] J. P. Perdew et al., Phys. Rev. B 46, 6671 (1992).

[21] P. E. Blöchl, Phys. Rev. B 50, 17953 (1994).

[22] The spectral broadening reflects a certain averaging over oxide regions with different Li content.

[23] B. K. Min, W. T. Wallace, A. K. Santra, and D. W. Goodman, J. Phys. Chem. B 108, 16339 (2004).

[24] J. Repp, G. Meyer, F. E. Olsson, and M. Persson, Science 305, 493 (2004). 